concentrations, and urinalysis were normal. Rheumatoid factor and antinuclear, antiextractable nuclear antigen, and antimitochondrial antibodies were absent. The LAC test was positive and low titre ( 12 arbitrary units) of aCL of the IgG isotype were found by enzyme linked immunoabsorbent assay. Both LAC and aCL tests were performed as previously described. ${ }^{4}$ A Doppler ultrasound examination of the lower limbs showed an extensive, bilateral thrombosis of both femoral and popliteal veins (figure). The child was discharged with weekly subcutaneous heparin calcium (Calciparine, Sanofi) and was then lost to follow up.

\section{Discussion}

We have reported a child with systemic onset JCA who developed an extensive deep vein thrombosis after a five month period of plaster immobilisation following a tibial fracture. When thrombosis was diagnosed, three months after plaster removal, both LAC and low titre IgG aCL were detected.

Only a few data exist on antiphospholipid antibodies in childhood. Our recent studies have shown a high frequency of aCL in children with $\mathrm{JCA}^{2}$ but no $\mathrm{aCL}$ associated thrombotic events have been observed in these cases. Therefore, in JCA, the real predictive value of aCL antibodies for developing thromboembolic events is weak, and in no way is antithrombotic prophylaxis indicated on the unique basis of a positive aCL assay.

It should be noted, however, that the clinical experience on aCL in adults has shown that associated thrombophilic factors such as smoking and use of oral contraceptives may enhance the risk of thrombosis. ${ }^{5}$ Prolonged immobilisation is well known to be associated with an increased risk of thromboembolism, and its association with antiphospholipid antibodies might have lead to deep vein thrombosis in our child.

This observation suggests that when children with JCA need immobilisation, such as after fractures of demineralised bones, the presence of antiphospholipid antibodies should be investigated as they may act as additional risk factors for thrombosis.

It should be noted that in our patient both LAC and $\mathrm{aCL}$ were detected and that it has been suggested that LAC represents a more specific, although less sensitive, marker of the thrombotic risk than aCL. ${ }^{6}$

In conclusion, our report suggests that short term prophylactic anticoagulant and/or antiaggregant treatment should be considered in those antiphospholipid antibody positive children with JCA who require immobilisation, particularly if circulating LAC is present.

1 Love PE, Santoro SA. Antiphospholipid antibodies, anticardiolipin and the lupus anticoagulant in systemic lupus erythematosus (SLE) and in non-SLE disorders. Prevalence and clinical significance. Ann Intem Med 1990,112.682-98.

2 Caporali R, Ravelli A, De Gennaro F, Neirotti G, Montecucco C, Martini A. Prevalence of anticardiolipin antibodies in juvenile chronic arthritis. Ann Rheum Dis 1991;50:599-601.

3 Montes de Oca M, Babron MC, Bletry $\mathrm{O}$, et al. Thrombosis in systemic lupus erythematosus: a French collaborative study. Arch Dis Child 1991;66:713-7.

4 Ravelli A, Caporali R, Bianchi E, et al. Anticardiolipin syndrome in childhood: a report of two cases. Clin Exp Rheumatol 1990;8:95-8.

5 Asherson RA, Harris EN, Hughes GRV. Complication of oral contraceptives and antiphospholipid antibodies. Arthrtis Rheum 1988;31:575-6.

6 Mackworth-Young C. Antiphospholipid antibodies: more than just a disease marker? Immunol Today 1990;11:605.

\title{
Severe anaemia and ileocolic anastomotic ulceration
}

\author{
A H Hamilton, J M Beck, G M Wilson, H J Heggarty, J W L Puntis
}

\section{Abstract}

Two children are described with anaemia from ileocolic anastomosic ulceration as a late complication of surgery in the newborn period. The anastomosis was revised in each case but in one child there was early recurrence of ulceration.

\section{(Arch Dis Child 1992;67:1385-6)}

Ulceration at the site of an ileocolic anastomosis as a late complication of neonatal surgery has been described, but is probably underrecognised. Rational management is hampered by lack of understanding of the underlying aetiology of the condition. We report our experience in two children with this unusual problem.

\section{Case reports}

CASE 1

A boy of 25 weeks' gestation required ventilation for respiratory distress, but after extubation he was established on full enteral feeds. At 9 weeks of age he developed abdominal distension and bloody diarrhoea and an abdominal $x$ ray film confirmed necrotising enterocolitis. Despite medical management his condition deteriorated, necessitating laparotomy. A diseased segment of ileum was resected and a cutaneous enterostomy fashioned, after which he made a good recovery. Two months later radiological investigation confirmed two colonic strictures that were resected and an ileocolic anastomosis performed. He was discharged home at 6 months of age, on full enteral feeding, and followed up at his local hospital.

He was referred to the surgical unit at the age of 7 years for investigation of anaemia. He had no symptoms, was on the third centile for both height and weight, and was passing two normal stools each day. Despite recent transfusion, investigation revealed a microcytic hypochromic anaemia, with a haemoglobin concentration of $65 \mathrm{~g} / \mathrm{l}$. Testing for faecal occult blood gave a strongly positive result. An upper gastrointestinal endoscopy and barium meal both gave normal results, but at colonoscopy the mucosa 
at the site of the ileocolic anastomosis was seen to be reddened and friable. $A^{99 m}$ Tc-labelled red cell scintiscan suggested bleeding was occurring from this region. At laparotomy, perianastomotic ulceration involving both small bowel and colonic mucosa was confirmed. There was no evidence of stricture. The ulcerated segment of bowel was resected and the anastomosis revised. Histological examination revealed non-specific ulceration.

Three months later his haemoglobin concentration fell to $60 \mathrm{~g} / \mathrm{l}$; colonoscopy showed fresh bleeding from recurrent ulceration at the site of the revised anastomosis. Treatment for six weeks in turn with oral sulphasalazine $40 \mathrm{mg} /$ $\mathrm{kg} /$ day, sucralfate $40 \mathrm{mg} / \mathrm{kg} /$ day, mesalazine 40 $\mathrm{mg} / \mathrm{kg} /$ day, and ranitidine $30 \mathrm{mg} / \mathrm{kg} /$ day failed to stop bleeding. The anastomosis was shown radiologically to be inaccessible to steroid enema.

Serum gastrin concentration was not raised (26 pmol/1, reference range $<40$ ); a hydrogen breath test was negative and empirical treatment for bacterial overgrowth with two weeks of metronidazole and trimethoprim had no influence on further blood loss. Over a six month period he required transfusion every three weeks. After discussion with parents it was decided that an ileostomy might stop blood loss and improve quality of life, and this was subsequently performed. Over the next 12 months his haemoglobin was maintained at a normal concentration, although large volume ileostomy losses were an unforeseen complication.

\section{CASE 2}

A boy was born at term with gastroschisis. Primary closure was not possible and part of the bowel became necrotic. Altogether $50 \%$ of the small bowel was resected and an ileocolic anastomosis fashioned. The gastroschisis was eventually closed on day 10 , with a porcine dermis patch. He was discharged home at the age of 9 weeks on a modular feed based on comminuted chicken. By the end of the first year of life he was tolerating soya milk and a variety of solids, although his weight gain was poor and stools continued to be loose.

At the age of 5 years he presented with bouts of abdominal pain and melaena. Investigation revealed a haemoglobin concentration of $50 \mathrm{~g} / 1$ with an iron deficient picture. The anaemia recurred despite transfusion and he was referred back to the surgical unit for investigation. The following studies gave normal results: upper gastrointestinal endoscopy, colonoscopy, contrast enema, radioisotope scan for Meckel's diverticulum, and ${ }^{99 m}$ Tc-labelled red cell scintiscan. Serum gastrin concentration was normal $(38 \mathrm{pmol} / \mathrm{l})$. Treatment with cimetidine $20 \mathrm{mg} / \mathrm{kg} /$ day for two months did not prevent anaemia. He underwent laparotomy and chronic ulceration was discovered at the site of the ileocolic anastomosis. There was no evidence of stricture and the anastomosis was revised; histology showed non-specific usceration. Eighteen months later he remains well with no recurrence of gastrointestinal blood loss.

\section{Discussion}

Ileocolic anastomosis may be necessary in necrotising enterocolitis, intussusception, or congenital abnormalities of the bowel. Extensive resection of ileum sometimes results in diarrhoea and malnutrition particularly if the ileocaecal valve is lost, or if in combination with partial colectomy. ${ }^{1}$ Perianastomotic ulceration appears to be a rare complication of ileocolic anastomosis in the newborn period presenting with severe anaemia up to 12 years after surgery. ${ }^{2}$ Colonoscopy is the most helpful investigation. The aetiology of the ulceration remains obscure and rational treatment problematic. Histological appearances give no clue to underlying mechanisms. Ulceration has not been related consistently to either anastomotic stricture or type of suture material. Blind loop syndrome with small bowel bacterial overgrowth and deconjugation of bile salts has been linked with mucosal ulceration. ${ }^{4}$ Classical symptoms include chronic obstruction, malnutrition, and malabsorption leading to growth failure. ${ }^{5}$ Both our patients were growing normally and neither was found to have blind loops at operation. On the basis that local factors such as bowel flora or $\mathrm{pH}$ might be playing a part, we gave treatment with broad spectrum antibiotics and $\mathrm{H}_{2}$ blockers, but with no cessation of bleeding. Although it has been reported that sulphasalazine can prevent bleeding, ${ }^{1}$ no such effect was seen in our patient given anti-inflammatory agents. Due to the severity of bleeding and requirement for repeated transfusions we chose revision of the ileocolic anastomosis. Unfortunately, recurrent ulceration rapidly followed, a complication previously described. ${ }^{3}$ An ileostomy successfully stopped bleeding but large volumes of effluent have created a new problem suggesting that the remaining small bowel is predominantly jejunum. ${ }^{6}$ Anastomotic revision appears to have been more successful in our second case.

If absence of the ileocaecal valve is a critical factor in aetiology perhaps the development of surgical techniques aimed at reconstruction of valve mechanism might offer a hope of cure. Unfortunately, the presence of a valve does not altogether preclude the possibility of anastomotic ulceration. ${ }^{3}$ A rational approach to management must depend on a future understanding of the aetiological mechanisms concerned, but long term follow up of children who have undergone ileocolic anastomosis in the newborn period is clearly essential.

\footnotetext{
1 Cosnes J, Gendre JP, le Quintrec Y. Role of the ileocaecal valve and site of intestinal resection in malabsorption after extensive small bowel resection. Digestion 1978;18:329-36.

2 Parashar K, Kyawhla S, Booth IW, Buick RG, Corkery JJ Ileocolic ulceration: a long term complication following ileocolic anastomosis. F Pediatr Surg 1988;23:226-8.

3 Couper RTL, Dury PR, Stafford SE, Filla RM, Marcon MA, Forstner GG. Late gastro-intestinal bleeding and protein loss after distal small bowel resection in infancy. $\mathcal{J}$ Pediatr Gastroenterol Nutr 1989;9:454-60.

4 Challacombe DN, Richardson JM, Edkins S, Hay IF. Ileal blind loop in childhood. Am $\mathcal{J}$ Dis Child 1974;128:719-23.

5 Bayes BJ, Hamilton JR. Blind loop syndrome in children. Arch Dis Child 1969;44:76-81.

6 David GR, Santa Ana CA, Morawski SG, Fordtran JS Permeability characteristics of human jejunum, ileum proximal colon and distal colon: results of potentia difference measurements and unidirectional fluxes. Gastroenterology 1982;83:844-50.
} 